\title{
Volatile elements in chondrites
}

\author{
N. BRAUKMÜLLER ${ }^{1,2 *}$, C. FUNK ${ }^{1}$, C. MÜNKER ${ }^{1}$, F. \\ WOMBACHER $^{1}$ \\ ${ }^{1}$ Institut für Mineralogie und Geologie, Universität zu Köln, \\ Germany \\ ${ }^{2}$ Institut für Geologische Wissenschaften, Freie Universität
} Berlin, Germany (n.braukmueller@fu-berlin.de)

Relative to CI chondrites, all differentiated and undifferentiated inner solar system materials are depleted in volatile elements such as $\mathrm{Zn}$, In and S [1]. Well constrained volatile element abundance patterns for chondrites are required to clarify primary solar nebular and secondary parent body volatile element fractionation processes and for comparison with differentiated planetary bodies like Earth.

Here we present high precision isotope dilution ICP-MS data for 11 volatile elements $(\mathrm{Cu}, \mathrm{Ga}, \mathrm{Ag}, \mathrm{Zn}, \mathrm{Te}, \mathrm{Sn}, \mathrm{Se}, \mathrm{S}$, $\mathrm{Cd}, \mathrm{In}, \mathrm{Tl}$ ) in mostly unequilibrated $\mathrm{OC}, \mathrm{EC}, \mathrm{CC}$ and Rumuruti chondrites (RC).

When plotted in order of decreasing $50 \%$ condensation temperature, our data confirm the previously detected "hockey stick" volatile element depletion pattern for CCs with $\mathrm{Cu}, \mathrm{Ga}$ and sometimes $\mathrm{Ag}$ belonging to the slope volatile elements, while the more volatile elements form an abundance plateau relative to CI $[2,3]$. This pattern has been related to primary volatile element depletion in the solar nebula, with plateau volatile element contents being related to matrix abundances $[3,4]$. Our new data suggests that primary volatile element depletion in RCs and OCs also resulted in a "hockey stick" pattern. However, the plateau is considerably disturbed for the most volatile elements ( $\mathrm{Se}$ $\mathrm{T} 1)$ which are heterogeneously distributed in the $\mathrm{RC}$ and $\mathrm{OC}$ parent bodies. This scatter and the heterogeneous distribution of $\mathrm{Cd}$ in $\mathrm{CV}$ and $\mathrm{CO}$ chondrites is attributed to secondary evaporation and recondensation processes on the meteorite parent bodies [5].

In ECs no clear correlation of elemental abundance with volatility is observed and no plateau pattern was identified. EH chondrites are less depleted than EL and EH4 are even enriched in $\mathrm{Cu}-\mathrm{Se}$ relative to CI. These complex depletion patterns indicate a complicated condensation-evaporation history in a highly reducing environment likely accompanied by sulfide and Fe-metal fractionation [6].

[1] Davis (2006) MESS, 295-307; [2] Takahashi et al. (1978) GCA 42, 97-106; [3] Braukmüller et al. (2018) GCA 239, 17-48, [4] Jacquet et al. (2016) MAPS 51, 851-869; [5] Wombacher et al. 2008, GCA 72, 646-667; [6] Alexander (2019) GCA 254, 246-276. 